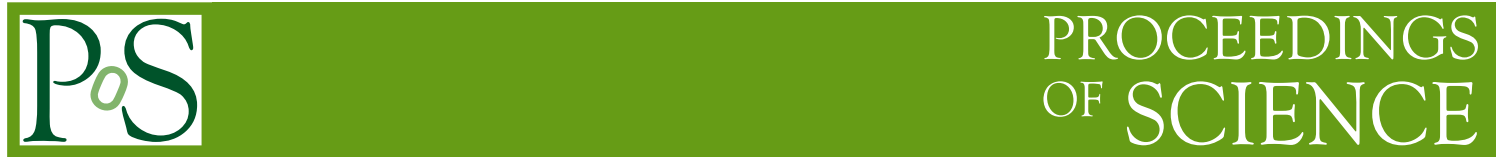

\title{
Gamma-ray Bursts as Probes of the First Stars
}

\section{Volker Bromm*}

Department of Astronomy, University of Texas at Austin, U.S.A.

E-mail: vbrommeastro.as.utexas.edu

We briefly review the basic theoretical framework for the formation of the first stars and galaxies. High-redshift gamma-ray bursts are among the most promising probes of this crucial epoch of first light. Utilizing GRBs, we will learn about the properties of individual first star progenitors, the cosmic star formation rate density at high redshifts, and the ionization structure and metal enrichment of the early intergalactic medium. Upcoming or planned missions, dedicated to the search of high-redshift bursts, promise to unlock their great potential in elucidating star and galaxy formation in the first billion years.

Gamma-Ray Bursts 2012 Conference -GRB2012,

May 07-11, 2012

Munich, Germany

\footnotetext{
* Speaker.
} 


\section{Introduction}

Understanding how the formation of the first stars and galaxies ended the cosmic dark ages is one of the key challenges in modern astronomy [2, 9, 28]. Within current $\Lambda \mathrm{CDM}$ cosmology, this moment of first light is predicted to occur at redshifts $z \simeq 20-30$, when the primordial gas is able to collapse into dark matter minihalos of mass $\sim 10^{6} \mathrm{M}_{\odot}$. Primordial stars thus fundamentally transformed the universe through their production of hydrogen-ionizing photons, initiating the process of reionization, and the first heavy chemical elements. The character of this transition is governed by the properties of the first sources of light, such as the initial mass function (IMF) of the first, Population III (Pop III) stars [5], their rotation rates [35], as well as the luminosities, colors, and escape fractions of primordial galaxies [10].

The initial billion years of cosmic evolution constitute the final, remaining gap in our longstanding endeavor to trace the entire history of the universe. Upcoming facilities such as the James Webb Space Telescope (JWST), and the next generation of extremely-large telescopes on the ground (GMT, TMT, E-ELT) promise to open up a direct window into this formative period. Despite their exquisite sensitivity at near-IR wavelenghts, even these observatories may not be able to directly probe the first stars, unless they formed in massive clusters. The only opportunity to probe individual Pop III stars may be to catch them at the moment of their explosive death. This could involve extremely energetic supernova (SN) events, such as hypernovae or pair-instability $\mathrm{SNe}[23,31]$, or gamma-ray bursts (GRBs). The latter fate depends on whether Pop III stars could give rise to suitable collapsar progenitors, involving rapidly rotating massive stars [29]. Since Pop III stars are predicted to fulfill both requirements (see the discussion below), GRBs are expected to be prevalent at very high redshifts. Indeed, GRBs may play a key role in elucidating primordial star formation, as well as the properties of the early intergalactic medium (IGM), given their extreme intrinsic brigthness, both of the prompt $\gamma$-ray emission, as well as that of the prolonged afterglow.

A number of features render GRBs ideal probes of the epoch of first light [28]: (i) Traditional sources to observe the high- $z$ universe, such as quasars and Lyman- $\alpha$ emitting galaxies, severly suffer from the effects of cosmological dimming, whereas GRB afterglows, if observed at a fixed time after the trigger, exhibit nearly-flat infrared fluxes out to very high $z$. This counter-intuitive effect arises, because a fixed time interval in the observer frame translates into an increasingly early time in the source frame. Such earlier times in turn sample the rapidly decaying GRB lightcurve at the moment of maximal brightness, thus compensating for the cosmological dimming (increasing luminosity distance). (ii) In the hierarchical setting of cosmic structure formation, earlier times are dominated by lower-mass host systems. The massive hosts required for quasars and bright galaxies therefore are "dying out" at the highest redshifts. GRBs, on the other hand, mark the death of individual stars, which can form even in very low-mass systems. (iii) Finally, Pop III GRBs would provide very clean background sources to probe the early IGM. Again reflecting the low masses of their hosts, any proximity effect should be much reduced, as ionized bubbles are confined to the immediate vicinity of the Pop III system; the IGM would thus largely remain unperturbed. In addition, the featureless, broken power-law nature of GRB afterglow spectra enables us to easily discern any signature imprinted by absorption and emission events along a given line of sight (LOS). The outlook for GRB cosmology thus is bright. Future missions, such as JANUS, Lobster, or SVOM, promise to fully unleash its potential. 

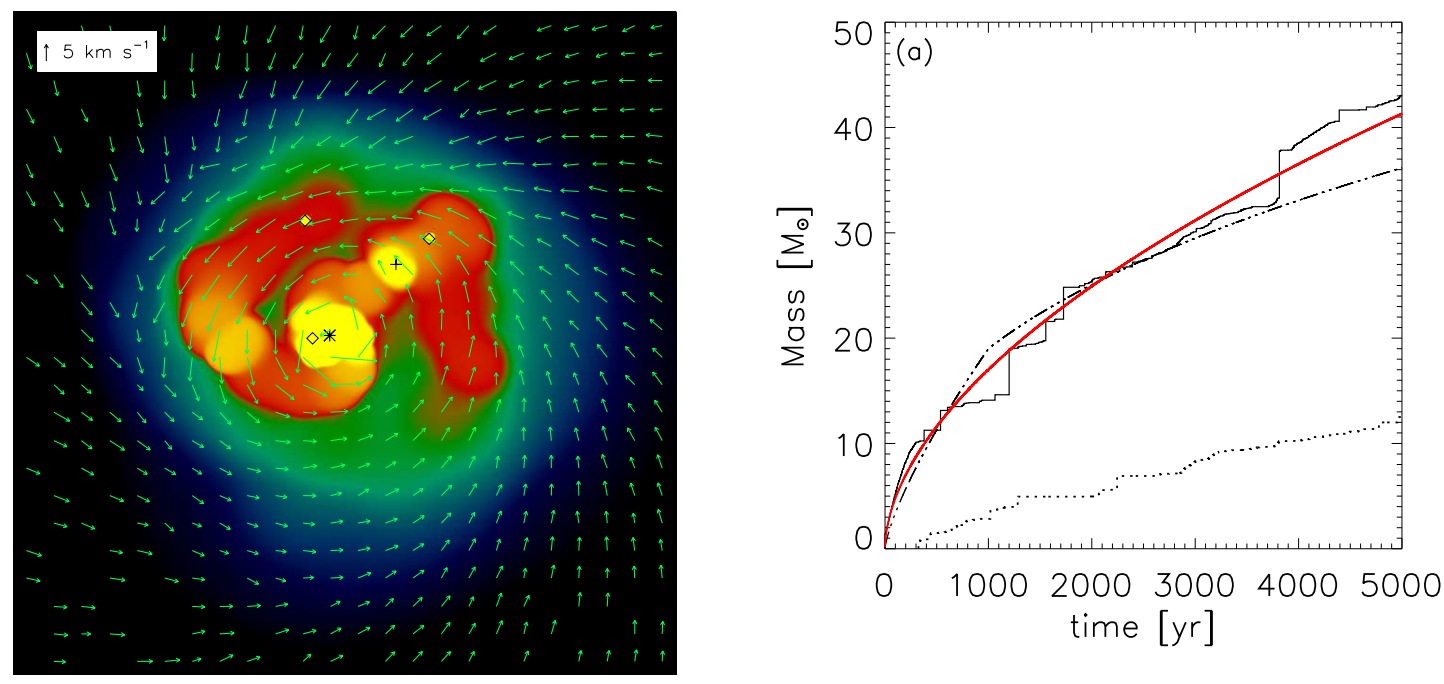

Figure 1: Simulating Pop III star formation (from [34]). Left panel: Multiple protostars embedded in an accretion disk. The colors symbolize the underlying density field (yellow marks highest density) within the central $5000 \mathrm{AU}$. Here, protostars are represented by sink particles, such that the asterisk marks the location of the most massive sink, the cross that of the second most massive one, and diamonds represent the other, smaller sinks. Shown is the situation $5000 \mathrm{yr}$ after initial sink formation. At this point, an ordered, nearly Keplerian velocity structure has been established within the disk. Right panel: Sink mass vs. time. The solid line shows the mass of the first sink particle, fitted by a power law according to $M \propto t^{0.55}$ (red line). The dash-dotted line depicts a reference case. The dotted line traces the mass growth of the second largest sink. As can be seen, sinks grow to masses $\gtrsim 10 \mathrm{M}_{\odot}$ within a few $1,000 \mathrm{yr}$.

In the following, we will briefly address some of the key questions posed by GRB cosmology: (i) Are Pop III stars suitable GRB progenitors? More precisely, does the primordial IMF include a range where black holes (BHs) are forming at the end of their life, and do (some) Pop III stars retain sufficient angular momentum prior to their death? (ii) How common were such Pop III bursts? (iii) How can we utilize any GRB at very high redshift to probe the state of the early IGM, regarding its degree of ionization and heavy-element enrichment?

\section{First Stars as GRB Progenitors}

The longstanding consensus view has been that the conditions in the early universe favored the formation of predominantly massive stars, such that the Pop III IMF was top-heavy $[1,4,5]$. This expectation rests on the much less efficient cooling in pure $\mathrm{H} / \mathrm{He}$ gas, where the only viable cooling agent is molecular hydrogen. The primordial gas can then cool to only about $\sim 200 \mathrm{~K}$, compared to the $10 \mathrm{~K}$ reached in dust-cooled molecular clouds in the present-day Milky Way. The correspondingly enhanced thermal pressure is reflected in a Jeans mass that is larger by one to two orders of magnitude in the Pop III case. Another element of this 'standard model' of primordial star formation has been that the first stars formed typically in isolation, one per minihalo.

Recently, beginning with work done in 2009, this traditional paradigm has been refined in important ways [40, 34, 13, 20, 21]. Supercomputing power, as well as algorithmic advances, now 
enable us to follow the protostellar collapse to densities where the initial hydrostatic core forms in the center of the cloud $\left(n \sim 10^{22} \mathrm{~cm}^{-3}\right)$ [41], and, crucially, beyond this stage into the main accretion phase. These simulations have demonstrated that accretion is mediated through a nearKeplerian disk. The hot conditions in the surrounding cloud result in extremely large rates of infall onto the disk $\left(\dot{M} \propto T^{3 / 2}\right)$; this rapid mass loading drives the disk inevitably towards gravitational instability, such that a small multiple of Pop III protostars emerges, often dominated by a binary system (see Fig. 1). It is not yet possible to push such $a b$ initio simulations all the way to the completion of the protostellar assembly process; the final mass of Pop III stars and their final IMF are thus still subject to considerable uncertainty. However, first attempts to carry out the radiation-hydrodynamical calculations required to treat the late accretion phase, where protostellar feedback tends to limit further infall, have confirmed the basic prediction: the first stars were typically massive, with masses of a few $\sim 10 \mathrm{M}_{\odot}$, although rarely very massive $\left(>100 \mathrm{M}_{\odot}\right)$, as previously thought, forming as a member of small multiple systems [30, 22, 36].

The first requirement for a collapsar central GRB engine, the emergence of BH remnants, is thus fulfilled. The binary nature of Pop III stars may also enable them, if the binary is sufficiently close to allow for Roche-lobe overflow and a common-envelope phase, to expel the extended hydrogen (and helium?) envelope. This may be crucial to prevent the quenching of the relativistic jet, triggered by the central engine $[8,37]$. What about the additional requirement that the collapsar progenitor retains enough angular momentum? This question ties in with the rate of rotation of Pop III stars, where almost nothing is known yet. A first attempt to address this within a fully cosmological context has recently been carried out [35], indicating that the first stars may have typically been very fast rotators, with surface rotation speeds of a few $10 \%$ of the break-up value. Such high rates of rotation would have important consequences for Pop III stellar evolution, possibly enabling strong mixing currents, and for the fate encountered at death. Thus, it is plausible that all requirements for a collapsar central engine were in place in the early universe. The next question now is: How common were Pop III GRBs, and do current or planned missions have a fair chance to detect them?

\section{High-Redshift GRB Rate}

It had long been realized that GRBs provide a powerful probe of the cosmic star formation history, extending out to very high redshifts where the first stars are expected to form $[26,6]$. Indeed, we now have examples of such bursts at very high redshifts, with the spectroscopically confirmed GRB 090423 at $z \simeq 8.2[32,38]$, and a photometrically constrained candidate at $z \sim$ 9.4 [14]. In addition, the radio afterglow of GRB 090423 has been detected with the VLA [12], providing useful constraints on the afterglow energetics and geometry, as well as on the circumburst density. From these observations, we have learned that the afterglow properties of the very high$z$ bursts are not significantly different from the more local sample. It remains, therefore, an open question how to uniquely identify any possible Pop III burst. Attempts to use features of the prompt $\gamma$-ray emission for this purpose may likely be ambiguous as well.

To explore the likely space of discovery, it is important to construct models of the high-redshift GRB rate. Schematically, this involves the following framework [8]: 

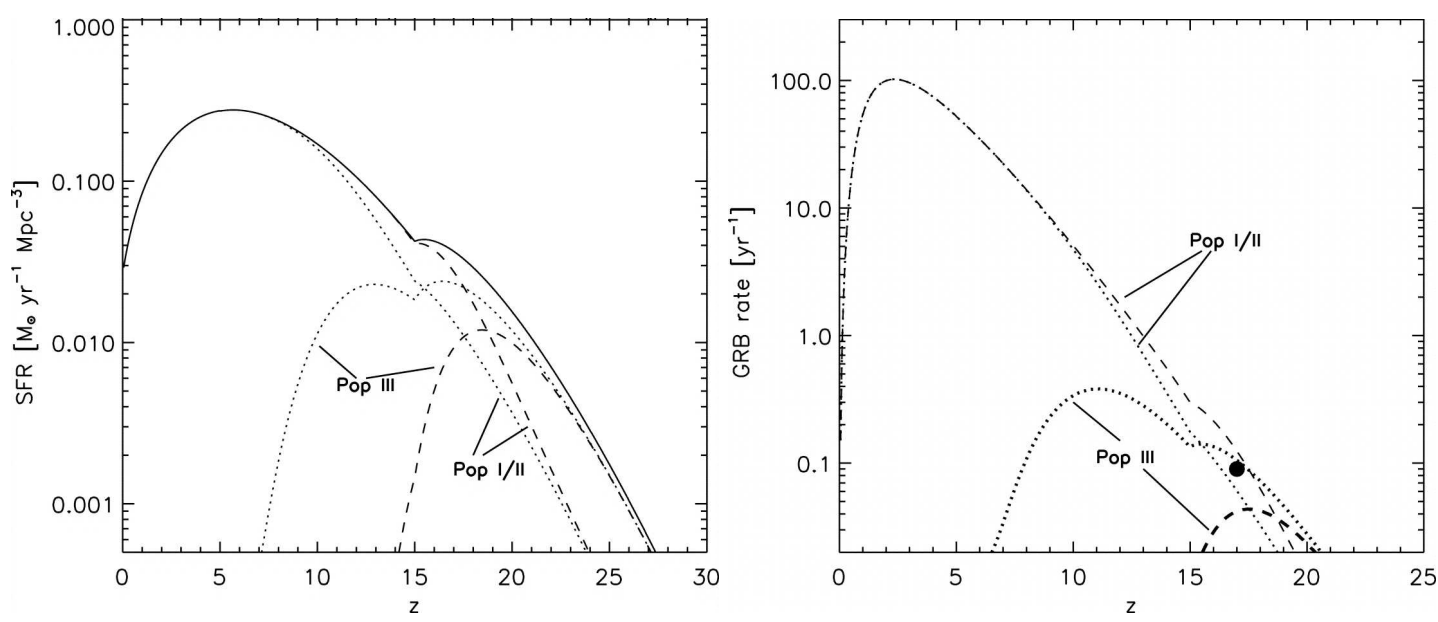

Figure 2: Predicting the Pop III GRB rate (from [8]). Left panel: Cosmic star formation rate density. The solid line represents the total SFRD, which is decomposed into a Pop I/II and Pop III mode, as marked. This decomposition depends on the strength of the chemical feedback, responsible for terminating primordial star formation (see text for further explanation). Here, the two cases shown correspond to weak (dotted line) and strong (dashed line) feedback. In the former case, Pop III star formation persists to much lower redshifts. Right panel: The predicted GRB rate vs. redshift, as observed with Swift. The decomposition into different modes mirrors that of the left panel. Again, the strength of the chemical feedback sets the level of a Pop III contribution in the sample, introducing considerable uncertainty into any prediction. The black dot marks the required degree of massive Pop III star formation, if reionization had occured already at $z \sim 17$; a scenario which is disfavoured by the most recent WMAP results.

$$
\frac{d N_{\mathrm{GRB}}^{\mathrm{obs}}}{d z}=\psi_{\mathrm{GRB}}^{\mathrm{obs}}(z) \frac{\Delta t_{\mathrm{obs}}}{(1+z)} \frac{d V}{d z},
$$

where $d N_{\mathrm{GRB}}^{\mathrm{obs}}$ is the number of GRBs, as observed with a given instrument, from within a reshift interval $d z, \psi_{\mathrm{GRB}}^{\mathrm{obs}}$ the number of bursts per comoving volume, and the other symbols have their usual meaning. The connection between the burst number density and cosmic star formation rate density (SFRD) can be expressed via:

$$
\psi_{\mathrm{GRB}}^{\mathrm{obb}}(z)=\eta_{\mathrm{GRB}} \psi_{*}(z) \int_{L_{\mathrm{lim}}(z)}^{\infty} p(L) d L,
$$

where $\psi_{*}(z)$ is the cosmic SFRD, $\eta_{\mathrm{GRB}}$ the GRB formation efficiency, $p(L)$ the GRB luminosity function, and $L_{\lim }(z)$ the minimum intrinsic luminosity required to detect the burst with a given instrument, from a given redshift (for details, see [8]).

Most of the intricacies come in when dealing with the efficiency factor. For simplicity, one could assign a constant value, possibly calibrating it to the observed Pop I/II value: $\eta_{\text {GRB }} \sim 10^{-9}$ bursts per unit solar mass [8]. Within such an idealized model, one typically estimates that of order $10 \%$ of all Swift GRBs should originate from $z>5$, with of order 0.1 Pop III bursts per year (see Fig. 2). Detection of a Pop III burst may thus lie just outside of the Swift capabilities, unless we are getting lucky. However, the real situation is likely much more complicated. The GRB efficiency could well depend on redshift, or on environmental factors, such as the metallicity of the host system [27]. Since the early modeling of the GRB redshift distribution, significant refinements 

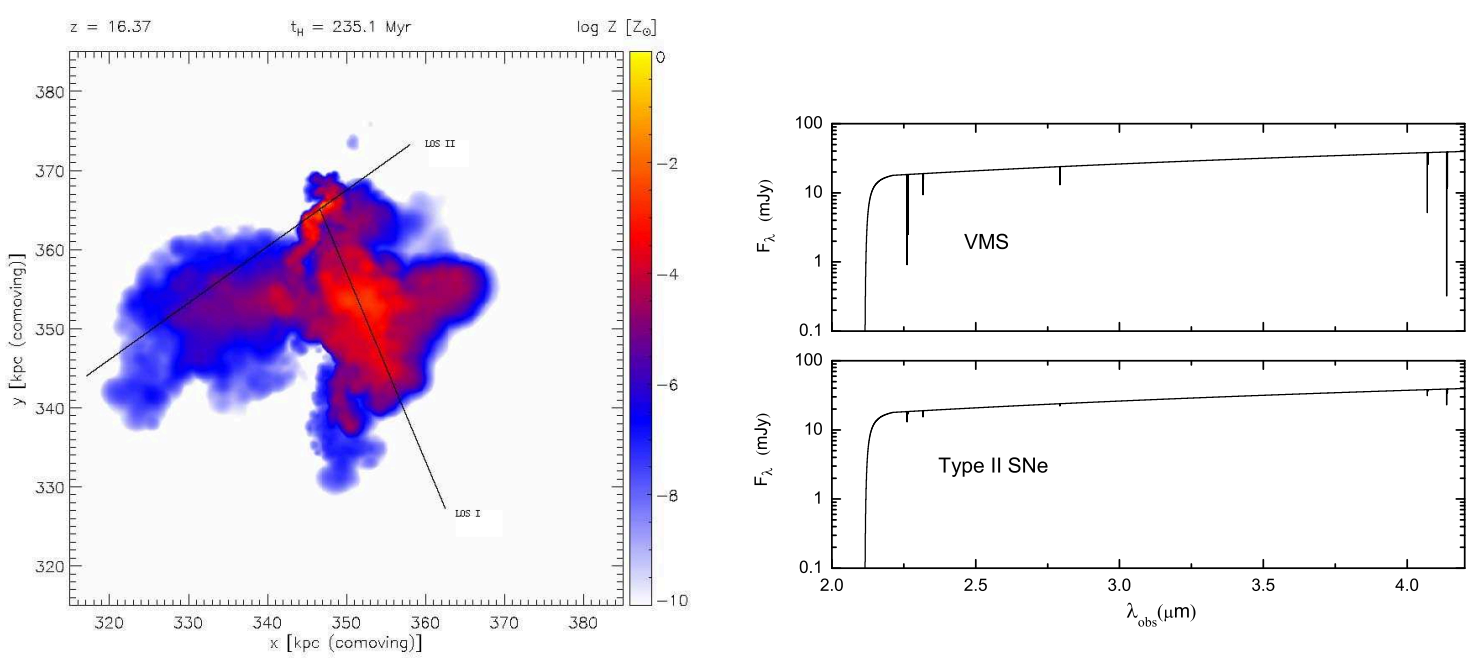

Figure 3: Probing pre-galactic metal enrichment (from [42]). Left panel: Metallicity distribution in a region of clustered Pop III star formation. The box size is $70 \mathrm{kpc}$ (comoving), shown at $z \simeq 16.4$. A Pop III GRB explodes at the intersection of the two lines. Right panel: Resulting near-IR absorption spectra. We consider two different cases for the Pop III SN enrichment, from conventional core-collapse (Type II) and from a very massive star (VMS) progenitor. The situation corresponds to 1 day (in the observer frame) after the GRB trigger. The lines are imprinted by low-ionization species of $\mathrm{C}, \mathrm{O}, \mathrm{Si}$, and $\mathrm{Fe}$. The sharp cutoff at lower wavelengths is due to the complete absorption of Ly $\alpha$ radiation in the neutral IGM. The VMS absorption-line signal should well be within the capabilities of the JWST.

have been added $[15,11,16,25,17]$. It is important, though, to not lose sight of the inherently very uncertain nature of this enterprise.

Among the most crucial uncertainties is the physical mechanism responsible for terminating the early mode of predominantly massive Pop III stars. Current thinking often posits that this Pop III to II transition is brought about by chemical feedback. The idea is that the cooling ability of star forming gas is greatly enhanced once it has been enriched with the first heavy elements beyond a threshold level, termed the 'critical metallicity' (of order $Z_{\text {crit }} \sim 10^{-4} Z_{\odot}$ ). The underlying physics is complex. Some models claim that fine-structure lines of neutral oxygen and singlyionized carbon may drive this transition [7]; others identify dust cooling as the key agent [33]. If dust were indeed responsible, predicted values of $Z_{\text {crit }}$ are typically smaller by one to two orders of magnitude, compared with the fine-structure scenarios.

\section{Probing the Intergalactic Medium}

Assuming standard, shocked-synchrotron theory, the properties of Pop III afterglows have been worked out $[18,24]$. Consistently, across a wide range of wavelengths, from the near-IR to radio, as well as in the X-ray bands, flux levels are predicted that bring such Pop III bursts within reach of existing and planned instruments. A key uncertainty in such modeling is what to assume for the circumburst density [42]. If we can identify these bursts through rapid follow-up in the near-IR, they will provide us with exquisite background sources to probe the early IGM. Firstly, we can place constraints on the ionized fraction of the high- $z$ IGM, as a function of redshift. This 
would provide a much more discerning picture of the cosmic reionization history, compared to the integral constraint from WMAP. In the latter case, by measuring the optical depth to Thomson scattering along the travel path of a CMB photon from the surface of last scattering to $z=0$, we cannot distinguish between models that can be quite different, but happen to yield the same LOS integral. The basic idea is to exploit the absorption strength in the red damping wing of the Lyman$\alpha$ resonance, which is very sensitive to any residual IGM neutral fraction [3]. This idea has been tested with the exquisite spectrum taken for GRB 050904 at $z \simeq 6.3$ [39]. The problem there proved to be the strong local column in neutral hydrogen, which completely overwhelmed any contribution from the general IGM. Again, the hope is that if we go to Pop III bursts, such local contamination would not be a problem, given that the first stars are exptected to form in low-mass host systems. Any local damping would then be small compared with the comological signal.

A second use of a Pop III GRB background source is to scrutinize the degree and nature of metal enrichment in the pre-galactic universe (see Fig. 3). The first stars are predicted to form in a highly biased region of the Gaussian random field of density fluctuations, such that their formation sites are strongy clustered. Any Pop III burst would then likely explode in a region that already may have been enriched by a small number of SNe [19]. The diagnostic provided by a high S/N near-IR spectrum of a Pop III afterglow may allow us to not only measure the overall metallicity at a given redshift. Additionally, we may also be able to distinguish between the abundance pattern from different kinds of explosion, such as a pair-instability SN, a hypernova, or a more conventional core-collapse (Type II) event [42].

\section{Acknowledgments}

I would like to thank the organizers for putting together a stimulating program, nicely conveying the excitement in this field. Support from NSF grant AST-1009928 and NASA ATFP grant NNX09AJ33G is gratefully acknowledged.

\section{References}

[1] T. Abel, G. L. Bryan and M. L. Norman, Science, 295, 93 (2002).

[2] R. Barkana and A. Loeb, Phys. Rep., 349, 125 (2001).

[3] —, Astrophys. J., 601, 64 (2004).

[4] V. Bromm, P. S. Coppi and R. B. Larson, Astrophys. J., 564, 23 (2002).

[5] V. Bromm and R. B. Larson, Annu. Rev. Astron. Astrophys., 42, 79 (2004).

[6] V. Bromm and A. Loeb, Astrophys. J., 575, 111 (2002).

[7] -, Nature, 425, 812 (2003).

[8] —, Astrophys. J., 642, 382 (2006).

[9] V. Bromm, N. Yoshida, L. Hernquist and C. F. McKee, Nature, 459, 49 (2009).

[10] V. Bromm and N. Yoshida, Annu. Rev. Astron. Astrophys., 49, 373 (2011).

[11] M. A. Campisi, U. Maio, R. Salvaterra and B. Ciardi, Mon. Not. R. Astron. Soc., 416, 2760 (2011). 
[12] P. Chandra et al., Astrophys. J., 712, L31 (2010).

[13] P. C. Clark, S. C. O. Glover, R. J. Smith, T. H. Greif, R. S. Klessen and V. Bromm, Science, 331, 1040 (2011).

[14] A. Cucchiara et al., Astrophys. J., 736, 7 (2011).

[15] F. Daigne, E. M. Rossi and R. Mochkovitch, Mon. Not. R. Astron. Soc., 372, 1034 (2006).

[16] R. S. de Souza, N. Yoshida and K. Ioka, Astron. Astrophys., 533, A32 (2011).

[17] J. Elliott, J. Greiner, S. Khochfar, P. Schady, J. L. Johnson and A. Rau, Astron. Astrophys., 539, A113 (2012).

[18] L. J. Gou, P. Mészáros, T. Abel, and B. Zhang, Astrophys. J., 604, 508 (2004).

[19] T. H. Greif, S. C. O. Glover, V. Bromm and R. S. Klessen, Mon. Not. R. Astron. Soc., 387, 1021 (2010).

[20] T. H. Greif, V. Springel, S. D. M. White, S. C. O. Glover, P. C. Clark, R. J. Smith, R. S. Klessen and V. Bromm, Astrophys. J., 737, 75 (2011).

[21] T. H. Greif, V. Bromm, P. C Clark, S. C. O. Glover, R. J. Smith, R. S. Klessen, N. Yoshida and V. Springel, Mon. Not. R. Astron. Soc., in press (arXiv:1202.5552) (2012).

[22] T. Hosokawa, K. Omukai, N. Yoshida and H. W. Yorke, Science, 334, 1250 (2011).

[23] J. Hummel, A. Pawlik, M. Milosavljevic and V. Bromm, Astrophys. J., in press (arXiv:1112.5207) (2012).

[24] S. Inoue, K. Omukai and B. Ciardi, Mon. Not. R. Astron. Soc., 380, 1715 (2007).

[25] E. E. O. Ishida, R. S. de Souza and A. Ferrara, Mon. Not. R. Astron. Soc., 418, 500 (2010).

[26] D. Q. Lamb and D. E. Reichart, Astrophys. J., 536, 1 (2000).

[27] N. Langer and C. A. Norman, Astrophys. J., 638, L63 (2006).

[28] A. Loeb, How did the first stars and galaxies form?, Princeton Univ. Press, Princeton (2010).

[29] A. I. MacFadyen and S. E. Woosley, Astrophys. J., 524, 262 (1999).

[30] C. F. McKee and J. C. Tan, Astrophys. J., 681, 771 (2008).

[31] T. Pan, D. Kasen and A. Loeb, Mon. Not. R. Astron. Soc., 422, 2701 (2012).

[32] R. Salvaterra et al., Nature, 461, 1258 (2009).

[33] R. Schneider, K. Omukai, A. K. Inoue and A. Ferrara, Mon. Not. R. Astron. Soc., 369, 1437 (2006).

[34] A. Stacy, T. H. Greif and V. Bromm, Mon. Not. R. Astron. Soc., 403, 45 (2010).

[35] A. Stacy, V. Bromm and A. Loeb, Mon. Not. R. Astron. Soc., 413, 543 (2011).

[36] A. Stacy, T. H. Greif and V. Bromm, Mon. Not. R. Astron. Soc., 422, 290 (2012).

[37] Y. Suwa and K. Ioka, Astrophys. J., 726, 107 (2011).

[38] N. R. Tanvir et al., Nature, 461, 1254 (2009).

[39] T. Totani et al., Publ. Astron. Soc. Japan, 58, 485 (2006).

[40] M. J. Turk, T. Abel and B. W. O’Shea, Science, 325, 601 (2009).

[41] N. Yoshida, K. Omukai and L. Hernquist, Science, 321, 669 (2008).

[42] F. Y. Wang, V. Bromm, T. H. Greif, A. Stacy, Z. G. Dai, A. Loeb and K. S. Cheng, Astrophys. J., submitted (2012). 